\title{
Developing Mathematical Models of Batteries in Modelica for Energy Storage Applications
}

\author{
Dr. Thanh-Son Dao Dr. Chad Schmitke \\ Maplesoft, Waterloo, Ontario, Canada, \{tdao, cschmitke $\}$ @maplesoft.com
}

\begin{abstract}
In this paper, effective and systematic steps in the mathematical modeling of high-fidelity battery models for simulating energy storage systems (ESS) will be presented. Two approaches to battery modeling will be discussed in this article: (1) the equivalent electrical circuit approach, and (2) the electrochemical approach. The battery models discussed in this article are developed based on the Modelica Standard Library specification 3.2.1 and commercialized as part of the Battery Component Library in MapleSim ${ }^{\circledR} 2015$.
\end{abstract}

Keywords: electrochemical, battery, Modelica, energy storage, electric vehicles, MapleSim

\section{Introduction}

Battery modeling is a challenging field that has been receiving a great amount of interest recently due to the great push from the portable electronic devices and the electric and hybrid electric vehicles (EV/HEV) industries. Despite of the differences in the power ranges and battery sizes in these applications, the two industries share a common goal: developing a new generation of batteries that allow devices to run for a longer period of time, while operating within a range that maximizes the battery's service life. In both of these areas, accurate and efficient battery modeling is vital to help maximize the performance of a device and its battery.

In this paper, we focus on the development of mathematical models of batteries, with an aim to produce high-fidelity battery models that are suitable for a wide range of applications. The battery models are implemented using the Modelica language, allowing them to be shared across multiple simulation platforms.

There have been several efforts in the past few years to commercialize Modelica-based battery component packages, including the battery libraries developed by AIT (Einhorn et al., 2011) and Modelon (Gerl et al., 2014; Modelon, 2015). Although some of the basic features of battery simulation such as battery voltage, state of charge (SOC), state of health (SOH), thermal effects, etc., are included in these packages, they face many limitations, which are mainly due to the equivalent electrical circuits approach used in generating their battery models. Some of the limitations include:

- It's not possible to maintain the battery voltage accuracy over a wide range of applied current: This is mainly because of the simplicity of the electrical circuit representations which do not allow them to describe the battery physics accurately under different operating conditions. For EV/HEV applications, this is a big obstacle as the charge/discharge current in an EV/HEV varies greatly during the operation of the vehicle and it is crucial that a battery model can portray this accurately.

- The thermal effects are simplified and neglect the changes in the entropy of the reaction of the electrode materials during each phase of the battery's operations. This is the most important heat source responsible for the reversible heat generation caused by the chemical reactions in the battery. As a result, the battery voltage and $\mathrm{SOH}$ cannot be simulated correctly as they are directly dependent on the battery temperature, making the battery components un-useful for battery parameter estimation and other applications that are temperature-dependent and require accurate simulation results. This includes studies and testing related to battery life and battery management systems (BMS).

- The circuit-based models are usually not scalable and are accurate only for the narrow ranges of specified parameter values. Any changes in the battery parameters such as capacity, thermal, applied current, etc., would cause large distortions in the simulation results.

In this article, two modeling approaches, namely the equivalent circuit approach, and the electrochemical approach are used to develop the battery models in MapleSim. All of the modeling issues mentioned above are either remedied or improved in the battery models presented in this article, depending on the modeling approach. The equivalent circuit models are upgraded based from the work (Chen and RincónMora, 2006). Several new features are added to the original representation, allowing the models to capture the thermal effects, degradation, and electrode 
chemistries precisely. This approach is described in details in Section 2. The electrochemical battery models are based on the porous electrode foundation proposed by (Newman and Tiedeman, 1975; Doyle et al., 1996). The symbolic and numerical techniques for handling the electrochemical battery equations and incorporating the equations into MapleSim are thoroughly discussed in Section 3. Section 4 and Section 5 discuss thermal effects and battery degradation, while Section 6 presents the structure of the Modelica implementation of the models in MapleSim. The battery library includes a parameter estimation worksheet programmed in Maple to allow the users to validate the battery parameters based on experiment measurements. Section 7 provides information about this, and examples outlining some key applications of battery models are presented in Section 8.

\section{Equivalent electrical circuit models}

Circuit-based models attempt to model the electrochemical physics of a battery using only electrical components. These component models can easily be incorporated into any system models, and are generally not computationally expensive.

Although the circuit models abstract away the electrochemical physics happening in the battery, circuit-based models are close to mapping the physics onto circuit elements.

The base structure for the equivalent-circuit models for lead-acid, nickel-metal hydride, and lithium-ion cells in MapleSim was developed based on the work of (Chen and Rincón-Mora, 2006). The internal structure of the model is shown in Figure 1. There are two main linear circuits in the diagram: (1) the RC circuit at the bottom models the instantaneous, short, and long-time responses of the battery; and (2) the large capacitor at the top models the battery capacity.

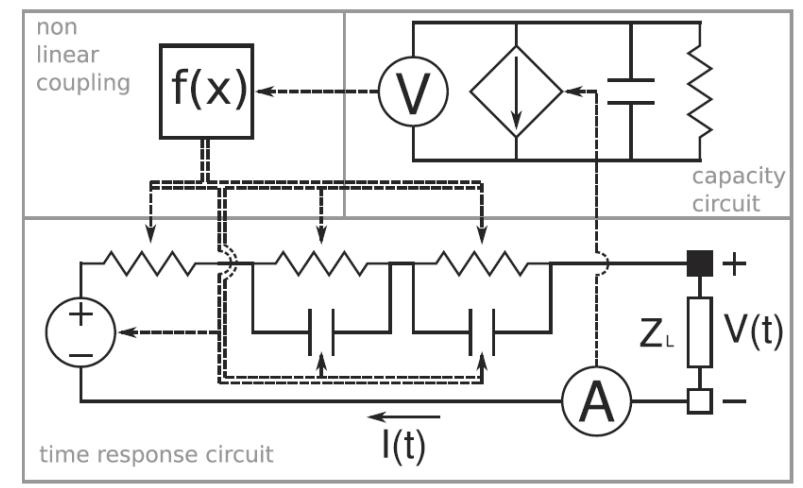

Figure 1. Schematic diagram of an equivalent circuit battery model.

The two circuits are non-linearly coupled using the following equations which describe the battery behaviors as the function of the battery SOC:

$$
\begin{aligned}
& C_{\text {cap }}=p_{1} \\
& V_{o c}=p_{2} \exp \left(p_{3} V_{s o c}\right)+p_{4}+p_{5} V_{s o c}+p_{6} V_{s o c}^{2} \\
& +p_{7} V_{s o c}^{3} \\
& \left.R_{\text {series }}=p_{8} \exp \left(p_{9} V_{\text {soc }}\right)+p_{10}\right) \\
& R_{\text {short }}=p_{11} \exp \left(p_{12} V_{\text {soc }}\right)+p_{13} \\
& C_{\text {short }}=p_{14} \exp \left(p_{15} V_{\text {soc }}\right)+p_{16} \\
& R_{\text {long }}=p_{17} \exp \left(p_{18} V_{\text {soc }}\right)+p_{19} \\
& C_{\text {long }}=p_{20} \exp \left(p_{21} V_{\text {soc }}\right)+p_{22}
\end{aligned}
$$

For all materials, the open-circuit potential (OCP) equation is curve-fitted based on experiment measurements. As an example, Figure 2 shows the OCP versus SOC for some materials of a lithium-ion battery. Example curve-fitted equations for the OCPs of $\mathrm{LiCoO}_{2}$ and graphite are shown in Equations (16) and (17) in Appendix A.

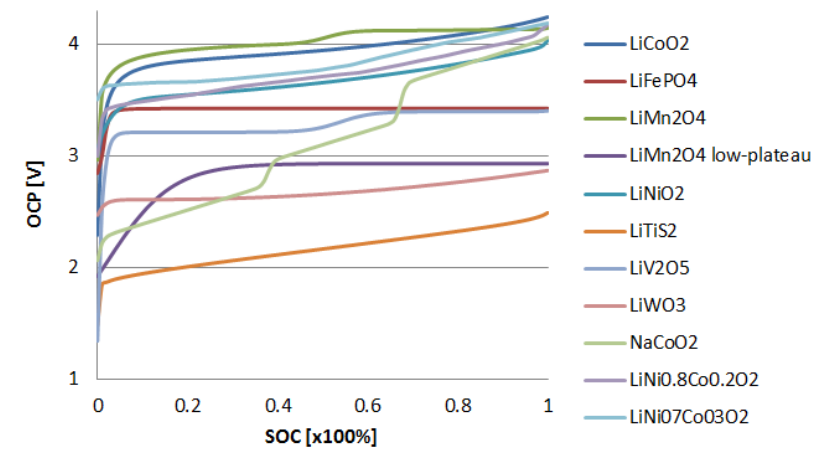

Figure 2. Open-circuit potentials for lithium-ion cathode materials.

\section{Electrochemical models}

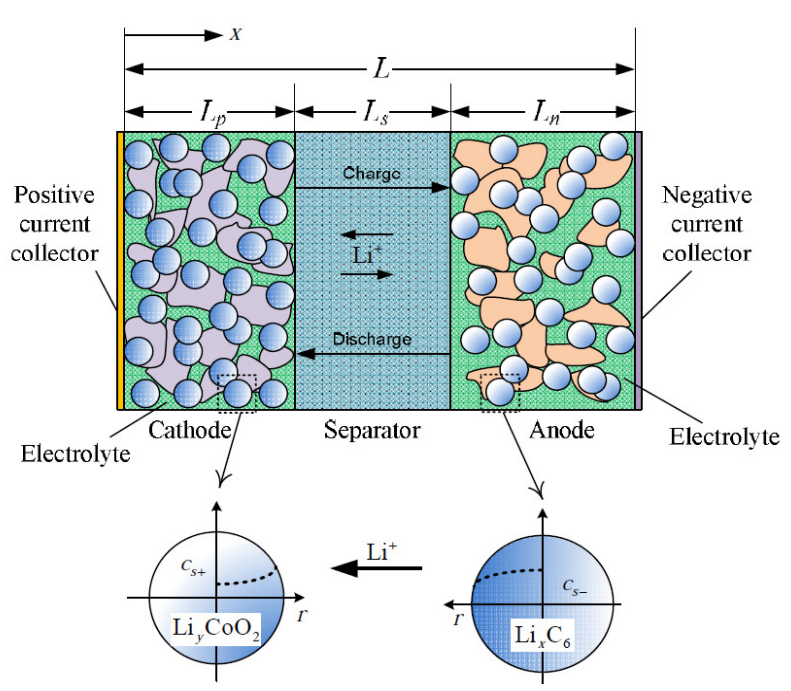

Figure 3. Anatomy of a lithium-ion cell (Dao et al., 2012).

Electrochemical models explicitly represent the chemical processes that take place in the battery. These types of models describe the battery physics in great details, making them the most accurate of all the battery models. 


\subsection{Governing equations}

Mathematical descriptions for electrochemical processes in most rechargeable cells are derived from the porous and concentrated solution theories proposed by (Newman and Tiedeman, 1975; Doyle et al., 1996) which mathematically describe charge/discharge and species transport in the solid and electrolyte phases across a spatial cell structure. The porous electrode theory uses partial differential equations (PDE) to describe the electrochemical processes. In general, these PDEs are derived based on Fick's law of diffusion for the active material concentration, Ohm's law for electrical potential distributions, and the Nernst and Butler-Volmer equations. In this article, the PDEs governing a lithium-ion cell in 1D spatial structure will be used as an example (see Figure 3).

The PDEs that describe the changes in $\mathrm{Li}+$ concentration in solid and liquid phases due to the gradient changes in the diffusive flow of $\mathrm{Li}+$ ions are described by:

$$
\frac{\partial c_{s}}{\partial t}=\frac{D_{s}}{r^{2}} \frac{\partial}{\partial r}\left(r^{2} \frac{\partial c_{s}}{\partial r}\right)
$$

and

$$
\epsilon \frac{\partial c_{e}}{\partial t}=\frac{\partial}{\partial x}\left(D_{e f f} \frac{\partial c_{e}}{\partial x}\right)+a\left(1+t^{+}\right) j
$$

Charge conservation in the solid phase of each electrode can be described by Ohm's law as

$$
\sigma \frac{\partial^{2} \Phi_{s}}{\partial x^{2}}=a F j
$$

Combining Kirchhoff's law with Ohm's law in the electrolyte phase yields:

$$
\begin{gathered}
-\sigma_{e f f} \frac{\partial \Phi_{s}}{\partial x}-\kappa_{e f f} \frac{\partial \Phi_{e}}{\partial x}+ \\
+\frac{2 \kappa_{e f f} R T}{F}\left(1-t^{+}\right) \frac{\partial \ln c_{e}}{\partial x}=I
\end{gathered}
$$

The Butler-Volmer equation describing the relationships between the current density, concentrations, and over-potential is given by:

$$
\begin{aligned}
& j=2 k\left(c_{s, \max }-c_{s, \text { surf }}\right)^{0.5}\left(c_{s, \text { surf }}\right)^{0.5} c_{e}^{0.5} \\
& \times\left[\exp \left(\frac{0.5 F}{R T} \mu\right)-\exp \left(-\frac{0.5 F}{R T} \mu\right)\right]
\end{aligned}
$$

These partial differential algebraic equations (PDAEs) are defined separately for each of the positive and negative electrode regions, and coupled with each other by the continuity in the boundary conditions. In total, 14 non-linear PDAEs are used to describe the behaviors of a lithium-ion cell. More details on battery equations can be found in (Newman et al., 1975; Doyle et al., 1996; Dao et al., 2012; Seaman et al., 2014)

\subsection{Model reduction}

The traditional methods for solving the battery PDAEs were mainly based on the classic finite difference techniques, which approximate continuous quantities as being constant within discrete evenly-spaced intervals along the spatial axis, $x$, and approximate the derivatives based on a Taylor series expansion. The end result is a large set of hundreds or even thousands of linear differential equations (DEs) which makes solving the whole battery system in real-time extremely difficult.

The approach for handling the battery PDEs implemented in MapleSim is different. Our approach is to use the symbolic computation strength of Maple $\AA$ 2015 to approximate the PDEs using Galerkin's method (Dao et al., 2012; Seaman et al., 2014) to produce fast simulating, yet accurate, models. The idea behind this method is to find the approximate numerical solution to a non-linear PDE using a set of orthogonal basis functions, and convert the PDE into a set of coupled ordinary differential equations (ODEs) based on temporal-spatial separation techniques. The resulting ODEs are small in size and are continuous, making the battery model they form more numerically stable and faster to solve.

Using the liquid phase concentration equation in Eq. (2) as an example, we start off with the spatial decomposition and allow time-varying coefficients as

$$
c_{e, a p p x}(x, t)=\sum_{j=1}^{N} \mu_{j}(t) \alpha_{j}(x)
$$

where $N$ is the number of node points, $\alpha_{j}(x)$ 's are the basis functions, and $\mu_{j}(t)$ 's are the unknown functions of time to be solved for. The basis functions, $\alpha_{j}(x)$ 's, must be orthogonal to ensure the resulting ODEs are linearly independent from each other.

Inserting approximate solution in (7) into the original PDE in (2) gives:

$$
\begin{aligned}
& R_{e}(x, t)=\epsilon \sum_{i=1}^{N} \alpha_{i}(x) \frac{\mathrm{d} \mu_{i}(t)}{\mathrm{d} t}+ \\
& -D_{\text {eff }} \sum_{i}^{N} \frac{\mathrm{d}^{2} \alpha_{i}(x)}{\mathrm{d} t^{2}} \mu_{i}(t)+a\left(1-t^{+}\right) j \approx 0
\end{aligned}
$$

This function is known as the residual. In Galerkin's method, we replace the condition that the residual should be approximately zero, with the condition that the residual should be orthogonal to the set of basis functions. The Galerkin techniques result in a set of ODEs which, when solved, give the approximate numerical solution, of the time-varying unknowns.

The reduced equations produced as the result of the Garlekin approach are then converted into Modelica to integrate with MapleSim using the Modelica component generation functionality in Maple.

\section{Thermal effects}

Modeling thermal effects in battery is crucial for many applications such as for testing and modeling the BMS, cooling system control, battery degradation, energy 
consumption in automotive/mobile devices, etc. Currently, several commercial libraries such as those developed by AIT and Modelon, model battery thermal behaviors by only looking at the irreversible heat generation due to the ohmic thermal loss caused by the battery's internal resistance. This approach neglects the most important heat source - the reversible heat due to the chemical reactions in the battery electrodes.

Thermal effects are incorporated in both electrochemical and equivalent circuit battery components using the following lumped thermal equation:

$$
\begin{aligned}
\rho V C_{p} \frac{\mathrm{d} T}{\mathrm{~d} t}=i\left(\mu_{p}\right. & \left.-\mu_{n}\right)+i T\left(\frac{\partial U_{p}}{\partial T}-\frac{\partial U_{n}}{\partial T}\right) \\
& +i^{2} R_{\text {int }}+h A_{\text {surf }}\left(T_{a}-T\right)
\end{aligned}
$$

In the above equation, $\frac{\partial U_{p}}{\partial T}$ and $\frac{\partial U_{n}}{\partial T}$ are the entropy of reaction which are dependent on the electrode materials.

The entropy of reaction for each electrode chemistry is measured from experiments and integrated into the battery components using a lookup table. The entropy of reaction curves for some of the chemistries for a lithiumion cell can be seen in Figure 4. MapleSim's lithium-ion cell component includes detailed electrochemical properties for 14 cathode chemistries and 3 anode chemistries, covering most of the commercially available lithium-ion electrode materials. The complete list of cathode and anode materials for lithium-ion cells supported by MapleSim 2015 is shown in Table 1 and Table 2 in Appendix B. The variety of the electrode materials allows the model to be used in different applications in both automotive and portable devices.

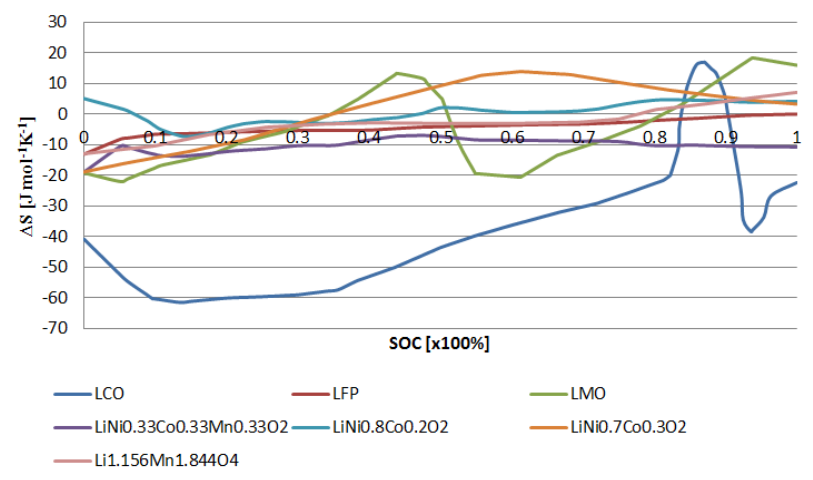

Figure 4. Entropy changes for lithium-ion cathode materials.

For all thermal models, the effects of cell temperature on the diffusion and ionic conductivity coefficients are modeled by Arrhenius' equation as:

$$
\begin{gathered}
D_{s}=D_{s, r e f} \exp \left[\frac{E_{d s}}{R}\left(\frac{1}{T_{\text {ref }}}-\frac{1}{T}\right)\right] \\
D_{e f f}=D_{e} \epsilon^{b r u g g} \exp \left[\frac{E_{d e}}{R}\left(\frac{1}{T_{r e f}}-\frac{1}{T}\right)\right] \\
\kappa_{e f f}=\kappa \exp \left[\frac{E_{k}}{R}\left(\frac{1}{T_{\text {ref }}}-\frac{1}{T}\right)\right]
\end{gathered}
$$

\section{Capacity fade}

The ability to model the degradation or capacity fade of rechargeable batteries during operation is critically important for predicting a battery's lifetime, and for designing and testing the BMS. In MapleSim, the battery life is modeled as the degradation caused by the formation of a solid-electrolyte interphase (SEI) layer in the negative electrode during charge (Pinson and Bazant, 2013). The following equation is used to describe the SEI thickness growth during charge:

$$
\frac{\mathrm{d} s}{\mathrm{~d} t}=\frac{k_{S E I} c_{e} M}{\left(1+\frac{k s}{D_{\text {diff }}}\right) \rho_{S E I}}
$$

Both the diffusion coefficient, $D_{\text {diff }}$, and the reaction rate, $k_{S E I}$, are temperature-dependent and can be described by Arrhenius' equation as:

$$
\begin{gathered}
D_{\text {diff }}=D_{0} \exp \left(-\frac{E_{a}}{R T}\right) \\
k_{S E I}=A_{e} \exp \left(-\frac{E_{a}}{R T}\right)
\end{gathered}
$$

The capacity fade is incorporated in both lithium-ion and NiMH cells for both electrochemical and equivalent circuit components based on the same degradation mechanism. The calculated battery $\mathrm{SOH}$ is dependent on the applied current, depth of discharge, temperature, and the cycling time. The degradation model also outputs the increase in the battery's internal resistance due to the formation of the SEI film.

$$
R_{S E I}=\frac{S}{\kappa}
$$

The parameters in the life model for lithium-ion cell have been estimated to closely fit the experiment measurements from a Lithium Iron Phosphate (LFP) found in (Liu et al., 2010). A comparison shows that the differences between simulation results and experiment measurements are small, as shown in Figure 5.

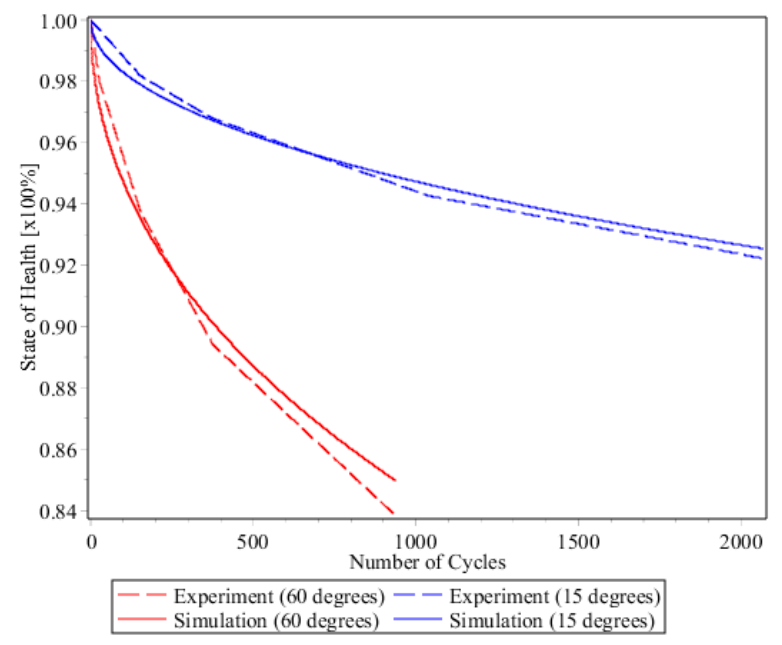

Figure 5. The life model (solid line) closely fits experiment data (dotted line) at $15^{\circ} \mathrm{C}$ and $60^{\circ} \mathrm{C}$. Data from (Liu et al., 2010) with $\mathrm{LiFePO}_{4}$ electrode. 
An example of the simulation results of a single lithium-ion cell being cycled for 20 hours at $80 \%$ Depth of Discharge (DOD) is shown in Figure 6.
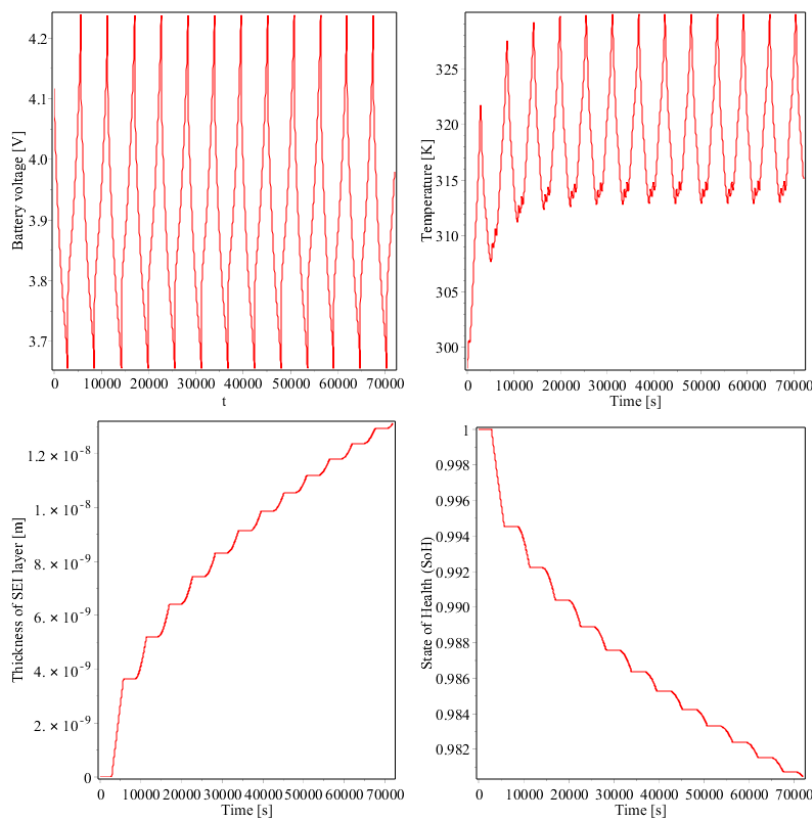

Figure 6. Degradation of $\mathrm{LiCoO}_{2}$ electrode after cycling for 20 hours at $80 \%$ DOD. Plots show battery voltage, temperature, thickness of SEI layer, and state of health.

\section{Modelica implementation}

The battery code has been implemented based on the Modelica Standard Library specification 3.2.1. The order-reduced battery equations were converted directly into Modelica using Maple commands.

\subsection{Single cell}

Both the electrochemical and equivalent circuit cell components are based on the following structure:

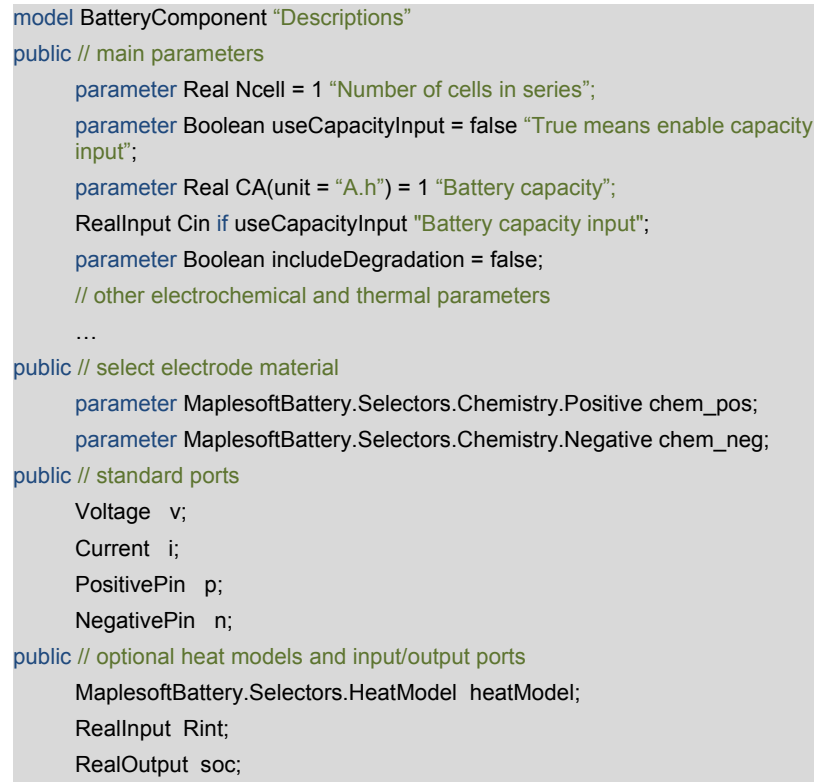

The selectors for cathode and anode chemistries, chem_pos and chem_neg, are implemented as dropdown menus as shown in Figure 7, allowing the user to select the electrode materials easily. A suitable model for the electrochemical behaviors and thermal effects of the cell will be used, depending on the choice of the chemistry. Additionally, the user can incorporate custom data for electrode chemistries and thermal properties based on input signal or lookup tables.

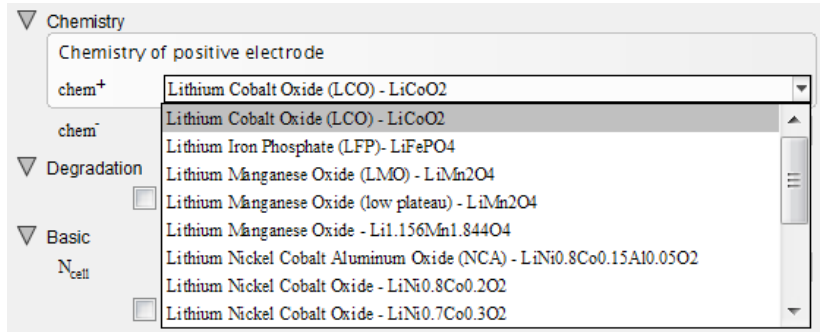

Figure 7. Lithium-ion cathode chemistry selector in MapleSim.

Thermal effects are also part of the battery components in MapleSim. There are three options for the thermal model: isothermal, convection cooling, and external heat port. The last option, external heat port, allows the other thermal components to be connected with the cell to simulate heat transfer between the cell and other parts of a system, such as heat transfer between the cells in a battery pack. If the convection cooling option is selected, the cell will exchange heat with an airflow through convection.

The battery degradation effects will be included in the simulation if the useCapacity Input option is selected. The battery model will output battery $\mathrm{SOH}$ in this case.

In addition to the main inputs/outputs, the user can turn on the input ports for the battery internal resistance and capacity, allowing these effects to be modeled based on a variable input or experiment measurements. Figure 8 shows a lithium-ion component with all the external ports turned on.

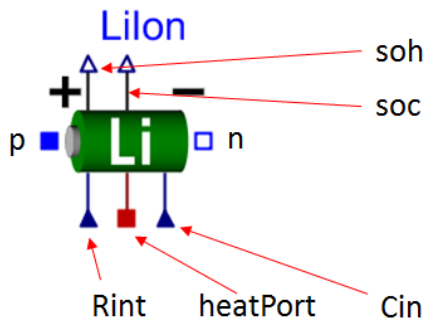

Figure 8. Ports of a battery component.

The battery components currently supported in MapleSim are shown in Figure 9. 


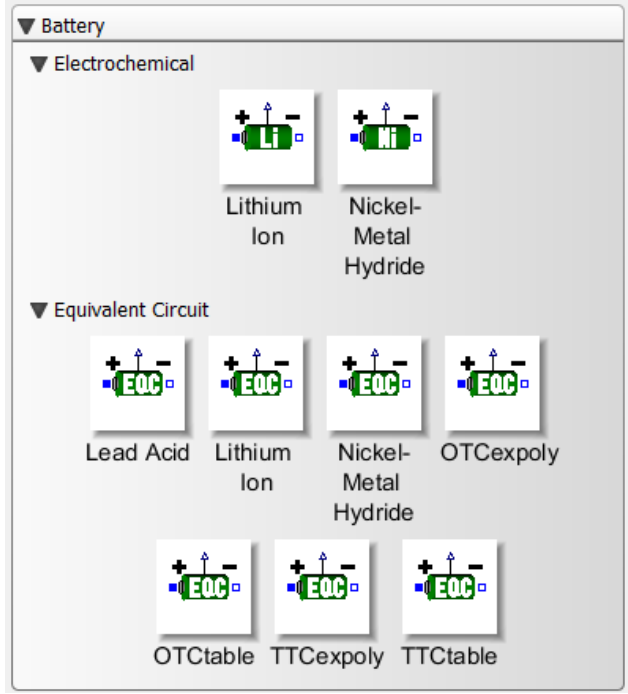

Figure 9. Battery components in MapleSim's battery component library.

\subsection{Stack}

The user can wire the individual cells to create a stack model easily. This can be done using MapleSim's advanced graphical user interface or based on standard Modelica descriptions. As an example, the Modelica script for $n$ lithium-ion cells wired in series as shown in Figure 10 is written as follows:

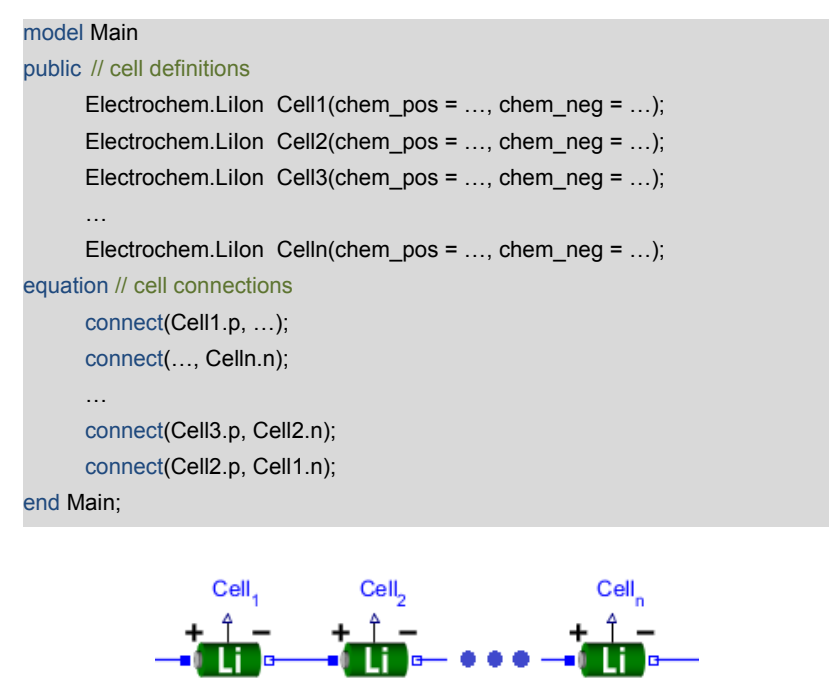

Figure 10. Lithium-ion cells connected in series.

\section{Battery parameter estimation}

The Battery Component Library in MapleSim includes worksheets programmed in the Maple language, for estimating battery parameters, based on experiment measurements.

The global optimization technique of Differential Evolution Algorithm is implemented in the worksheet to effectively perform the search for multiple parameters and find the best possible solution. This algorithm is part of the Global Optimization Toolbox in Maple and is powered by Optimus ${ }^{\circledR}$ technology from Noesis Solutions.

Figure 11 and Figure 12 show the parameter estimation results for a $\mathrm{LiCoO}_{2}$ (LCO) lithium-ion battery based on two sets of test data.

\begin{tabular}{|c|c|c|c|c|c|}
\hline Parameter & & Current & Minimum & & Maximum \\
\hline $\mathrm{CA}$ & $\nabla$ & 2.447 & .1 & 0 & 300 \\
\hline Ip & $\nabla$ & $0.7177 e-4$ & $0.10 e-4$ & 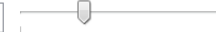 & $0.200 e^{-3}$ \\
\hline Ln & $\nabla$ & $0.8270 e-4$ & $0.10 e-4$ & -0 & $0.200 e-3$ \\
\hline ep & 目 & .3846 & .1 & 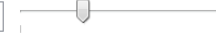 & .99 \\
\hline en & $\nabla$ & .3843 & .1 & 4 & .99 \\
\hline es & $\nabla$ & .7254 & .1 & $-7]$ & .99 \\
\hline efp & $\nabla$ & $0.2565 e-1$ & $0.1 \mathrm{e}-1$ & & .2 \\
\hline efn & V & $0.5127 e-1$ & $0.1 \mathrm{e}-1$ & & .2 \\
\hline Rsp & $\nabla$ & $0.1518 \mathrm{e}-5$ & $0.1 \mathrm{e}-5$ & H & $0.20 \mathrm{e}-4$ \\
\hline Rsn & $\nabla$ & $0.9849 e-5$ & $0.1 \mathrm{e}-5$ & 4 & $0.20 \mathrm{e}-4$ \\
\hline
\end{tabular}

Figure 11. Battery parameters used for curve fitting.

The plots in Figure 12 show that the simulation results closely resemble experiment data.

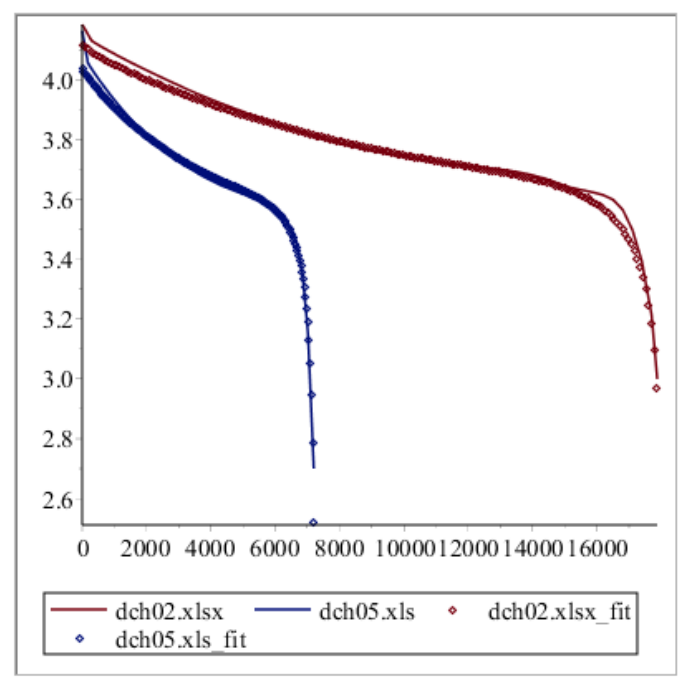

Figure 12. Simulation results (solid line) for battery parameter identification closely resemble experiment data (dotted line).

\section{Applications}

The structure and robustness of the battery components make them useful for many applications.

\section{Example 1:}

The example in Figure 13 shows an electric vehicle powered by a 30Ah Lithium Iron Phosphate (LFP) battery pack which has a maximum voltage of $390 \mathrm{~V}$ when fully charged. The LFP cathode and Lithium Titanate (LTO) anode are chosen for their good thermal stability. The model also features an asynchronous induction motor. The combined efficiency of the motor and power electronics is modeled using an efficiency map, which allows the 
simulated vehicle's energy consumption to be realistic. The battery pack is air cooled, using thermal parameters defined through a parameter block. The lithium-ion component outputs key information about the battery's operation such as SOC, temperature, voltage, and current values, as the vehicle follows the EPA highway drive cycle.

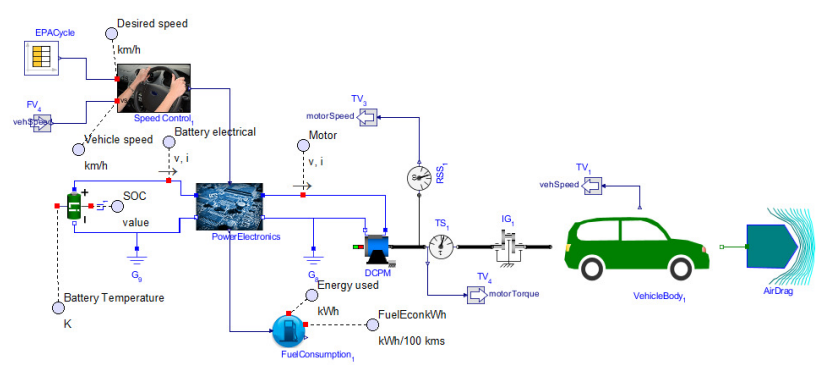

Figure 13. MapleSim model of an electric vehicle with an LFP lithium-ion battery.

The simulation results in Figure 14 show the vehicle speed and battery SOC. The controller does a good job in controlling the vehicle's speed. Depending on the direction of the vehicle's speed, the motor works in motoring mode. When the vehicle slows down, the motor becomes an electrical generator, which captures the brake energy to recharge the battery through regenerative braking.

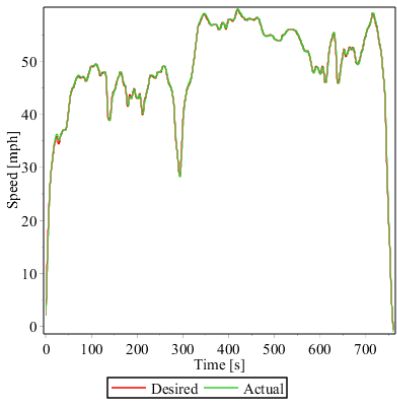

(a)

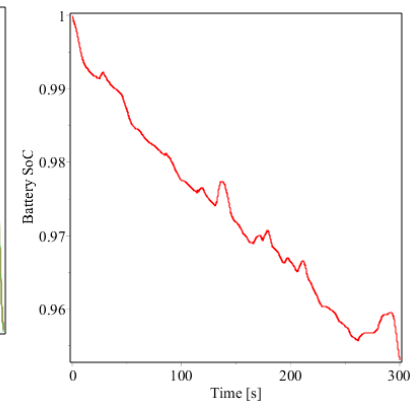

(b)
Figure 14. Vehicle speed (a) and battery SOC (b).

\section{Example 2:}

The following example (Figure 15) shows the simulation of the thermal exchange between lithiumion cells connected in a series/parallel structure. The 6 cells used in the model exchange heat between the adjacent cells that are in contact with them through thermal conductivity.

The differences in cell temperatures result in an imbalance in the cell voltages (i.e., some cells have lower voltages than the other cells), causing the currents through the series strings to vary (see Figure 16). In extreme cases, the "weak" cells (i.e., cells having lower voltages) can become over-heated due to short-circuiting and have to be remedied using a balancing current. The stack voltage is shown in Figure 17.

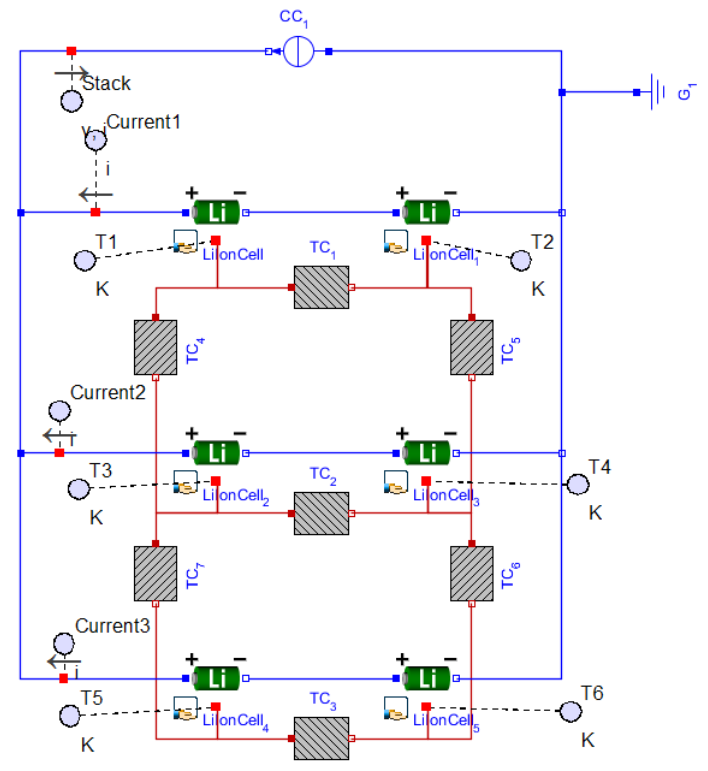

Figure 15. Thermal exchange in battery pack wired in series/parallel configuration.

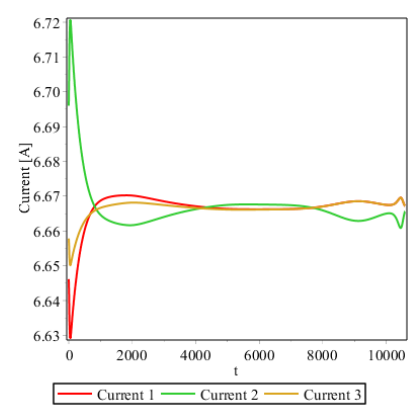

(a)

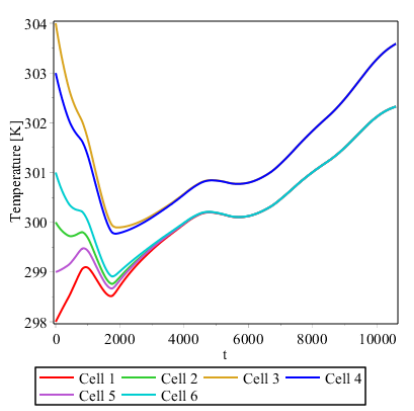

(b)
Figure 16. Current through each string of cells connected in series (a) and cell temperatures (b).

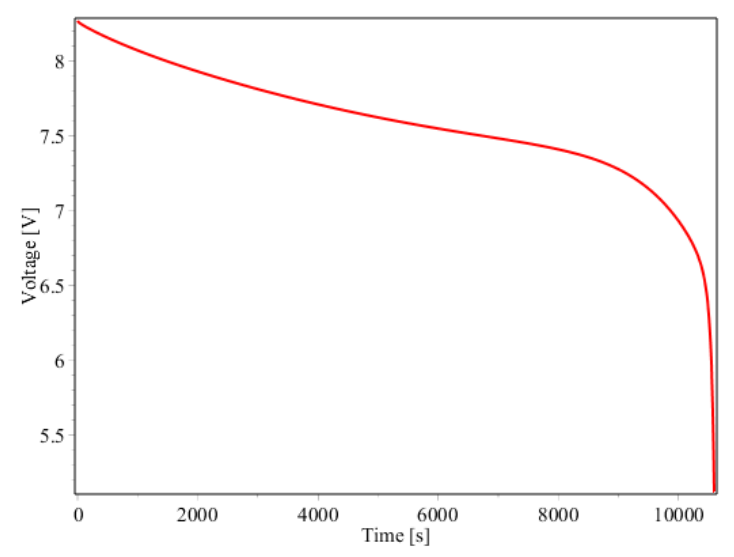

Figure 17. Stack voltage.

\section{Conclusion}

In this paper, we have shown two methods of generating battery model. While equivalent electrical models attempt to model the electrochemical physics of a battery using only electrical components, the 
resulting battery component, which is computationally inexpensive to incorporate into system models, has many limitations.

Electrochemical models on the other hand, are the most accurate because they describe the physics of a battery by explicitly representing the chemical processes that take place within it. Starting with PDEs to describe these electrochemical processes, symbolic techniques are then applied to approximate the PDEs using Galerkin's method, to arrive at the final set of reduced equations.

Capacity fade is incorporated into both the equivalent circuit and electrochemical battery components, with simulation results showing that battery life achieved by the components is a close fit with experiment measurements. The battery components also incorporate thermal effects, and include Maple language-based worksheets for battery parameter estimation, based on experiment measurements.

The battery components were implemented based on the Modelica Standard Library specification 3.2.1, and commercialized as part of the Battery Component Library in MapleSim ${ }^{\mathrm{TM}}$. The battery library also comes with a parameters identification worksheet which ensures a high level of fidelity in the battery components, making them suitable for a wide range of applications.

\section{References}

M. Chen and G. Rincón-Mora. Accurate Electrical Battery Model Capable of Predicting Runtime and I-V Performance. IEEE Trans. On Energy Conversion. 21(2):504-511, 2006.

T.-S. Dao, C.P. Vyasarayani, and J. McPhee. Simplification and Order Reduction of Lithium-Ion Battery Model Based on Porous-Electrode Theory. Journal of Power Sources, 198:329-337, 2012.

M. Doyle, J. Newman, C. Schmutz, and J.M. Tarascon. Comparison of Modeling Predictions with Experimental Data from Plastic Lithium Ion Cells. Journal of the Electrochemical Society, 143(6):1890-1903, 1996.

M. Einhorn, F.V. Conte, C. Niklas,, H. Popp, and J. Fleig. A Modelica Library for Simulation of Electric Energy Storages. The $8^{\text {th }}$ International Modelica Conference, 2011.

J. Gerl, L. Janczyk, I. Krueger, and N. Modrow. A Modelica Based Lithium Ion Battery Model. The $10^{\text {th }}$ International Modelica Conference, 2014.

P. Liu, J. Wang, J. Hicks-Garner, E. Sherman., S. Soukiazian, M. Verbrugge, H. Tataria, J. Musser, and P. Finamore. Aging Mechanisms of $\mathrm{LiFePO}_{4}$ Batteries Deduced by Electrochemical and Structural Analysis. Journal of the Electrochemical Society, 157(4):A499A507, 2010.

J. Newman and W. Tiedeman. Porous-Electrode Theory with Battery Applications. AIChE Journal, 21(1):25-44, 1975.
M.B. Pinson and M.Z. Bazant. Theory of SEI Formation in Rechargeable Batteries: Capacity Fade, Accelerated Aging, and Lifetime Prediction. Journal of the Electrochemical Society, 160(2):A243-A250, 2013.

A. Seaman, T.-S. Dao, and J. McPhee. A Survey of Mathematics-Based Equivalent-Circuit and Electrochemical Battery Models for Hybrid and Electric Vehicle Simulation. Journal of Power Sources. 256:410423, 2014.

Modelon Battery Library [Web]: http://www.modelon.com/products/modelicalibraries/battery-library-release-information/

\section{List of symbols}

\begin{tabular}{|c|c|}
\hline$a$ & specific surface area $\left[\mathrm{m}^{2} \mathrm{~m}^{-3}\right]$ \\
\hline$A_{e}$ & factor for reaction rate equation $\left[\mathrm{m} \mathrm{s}^{-1}\right]$ \\
\hline rugg & Bruggeman's coefficient \\
\hline$c_{e}$ & concentration in electrolyte phase $\left[\mathrm{mol} \mathrm{m}^{-3}\right]$ \\
\hline$c_{S}$ & concentration in solid phase $\left[\mathrm{mol} \mathrm{m}^{-3}\right]$ \\
\hline$c_{s, \max }$ & maximum concentration in solid phase $\left[\mathrm{mol} \mathrm{m}^{-3}\right]$ \\
\hline$c_{s, \text { surf }}$ & surface concentration in solid phase $\left[\mathrm{mol} \mathrm{m}^{-3}\right]$ \\
\hline$D_{0}$ & diffusion coefficient at standard conditions $\left[\mathrm{m}^{2} \mathrm{~s}^{-1}\right]$ \\
\hline$D_{s}$ & diffusion coefficient $\left[\mathrm{m}^{2} \mathrm{~s}^{-1}\right]$ \\
\hline$D_{s, r e f}$ & reference diffusion coefficient $\left[\mathrm{m}^{2} \mathrm{~s}^{-1}\right]$ \\
\hline$D_{e f f}$ & electrolyte diffusion coefficient $\left[\mathrm{m}^{2} \mathrm{~s}^{-1}\right]$ \\
\hline$E_{a}$ & activation energy $\left[\mathrm{J} \mathrm{mol}^{-1}\right]$ \\
\hline$E_{d e}$ & activation energy for diffusion $\left[\mathrm{J} \mathrm{mol}^{-1}\right]$ \\
\hline$E_{d s}$ & activation energy for diffusion $\left[\mathrm{J} \mathrm{mol}^{-1}\right]$ \\
\hline$E_{k}$ & activation energy for ionic conductivity $\left[\mathrm{J} \mathrm{mol}^{-1}\right]$ \\
\hline$I$ & applied current $[\mathrm{A}]$ \\
\hline$j$ & wall-flux of ions $\left[\mathrm{mol} \mathrm{m}^{2} \mathrm{~s}^{-1}\right]$ \\
\hline$k$ & reaction rate constant $\left[\mathrm{mol}\left(\mathrm{mol}^{-1} \mathrm{~m}^{3}\right)^{3 / 2}\right]$ \\
\hline$k_{S E I}$ & rate constant of SEI formation $\left[\mathrm{m} \mathrm{s}^{-1}\right]$ \\
\hline$M$ & molar mass of SEI layer $\left[\mathrm{kg} \mathrm{mol}^{-1}\right]$ \\
\hline$r$ & radius of intercalatin particle $[\mathrm{m}]$ \\
\hline$R$ & ideal gas constant $\left[\mathrm{J} \mathrm{K}^{-1} \mathrm{~mol}^{-1}\right]$ \\
\hline$s$ & thickness of SEI layer [m] \\
\hline$t$ & time, $[\mathrm{s}]$ \\
\hline$t^{+}$ & transference number in the electrolyte \\
\hline$T$ & battery temperature $[\mathrm{K}]$ \\
\hline$T_{\text {reff }}$ & temperature at standard conditions $[\mathrm{K}]$ \\
\hline$x$ & main dimension across the cell sandwich [m] \\
\hline$\alpha_{i}$ & basis function \\
\hline$\epsilon$ & porosity of electrode \\
\hline$\Phi_{s}$ & electrical potential in solide phase [V] \\
\hline$\Phi_{e}$ & electrical potential in electrolyte phase $[\mathrm{V}]$ \\
\hline$\kappa$ & Specific conductivity coefficien $\left[\mathrm{m} \mathrm{A}^{-1}\right]$ \\
\hline$\kappa_{e f f}$ & effective ionic conductivity of electrolyte $\left[\mathrm{S} \mathrm{m}^{-1}\right]$ \\
\hline$\mu$ & over-potential [V] \\
\hline$\mu_{i}$ & time-dependent variable of the $\mathrm{i}$-th basis function \\
\hline$\rho_{S E I}$ & density of SEI layer $\left[\mathrm{kg} \mathrm{m}^{-3}\right]$ \\
\hline$\sigma$ & electronic conductivity of in solid phase $\left[\mathrm{S} \mathrm{m}^{-1}\right]$ \\
\hline
\end{tabular}




\section{Appendix A}

OCP equations for $\mathrm{LiCoO}_{2}$ cathode and $\mathrm{LiC}_{6}$ anode:

$$
\begin{aligned}
U_{p}=(-3.234- & 638.136 \theta+2387.637 \theta^{2}-4027.7467 \theta^{3}+4106.484 \theta^{4} \\
& -2790.517 \theta^{5}+1292.901 \theta^{6}-401.8703962 \theta^{7}+79.910 \theta^{8} \\
& \left.-9.1764 \theta^{9}+0.463 \theta^{10}\right) /\left(-1.411-160.70 \theta+604.048 \theta^{2}\right. \\
& -1008.0656 \theta^{3}+1005.1301 \theta^{4}-663.87 \theta^{5}+299.134 \theta^{6}-90.922 \theta^{7} \\
& \left.+17.8147 \theta^{8}-2.032 \theta^{9}+0.1025 \theta^{10}\right)
\end{aligned}
$$

In both equations, $\theta$ indicates the battery SOC.

\section{Appendix B}

Table 1. List of cathode materials for Lithium-ion cells supported by MapleSim.

\begin{tabular}{|l|l|}
\hline Chemical composition & $\begin{array}{l}\text { Common name, } \\
\text { where given }\end{array}$ \\
\hline $\mathrm{LiCoO}_{2}$ & $\mathrm{LCO}$ \\
\hline $\mathrm{LiFePO}$ & $\mathrm{LFP}$ \\
\hline $\mathrm{LiMn}_{2} \mathrm{O}_{4}$ & $\mathrm{LMO}$ \\
\hline $\mathrm{LiMn}_{2} \mathrm{O}_{4}-$ low plateau & \\
\hline $\mathrm{Li}_{1.156} \mathrm{Mn}_{1.844} \mathrm{O}_{4}$ & \\
\hline $\mathrm{LiNi}_{0.8} \mathrm{Co}_{0.15} \mathrm{Al}_{0.05} \mathrm{O}_{2}$ & $\mathrm{NCA}$ \\
\hline $\mathrm{LiNi}_{0.8} \mathrm{Co}_{0.2} \mathrm{O}_{2}$ & \\
\hline $\mathrm{LiNi}_{0.7} \mathrm{Co}_{0.3} \mathrm{O}_{2}$ & \\
\hline $\mathrm{LiNi}_{0.33} \mathrm{Mn}_{0.33} \mathrm{Co}_{0.33} \mathrm{O}_{2}$ & $\mathrm{NMC}$ \\
\hline $\mathrm{LiNiO}_{2}$ & \\
\hline $\mathrm{LiTiS}_{2}$ & \\
\hline $\mathrm{LiV}_{2} \mathrm{O}_{5}$ & \\
\hline $\mathrm{LiWO}_{3}$ & \\
\hline $\mathrm{NaCoO}_{2}$ & \\
\hline
\end{tabular}

Table 2. List of anode materials for Lithium-ion cells supported by MapleSim.

\begin{tabular}{|l|l|}
\hline Chemical composition & $\begin{array}{l}\text { Common name, } \\
\text { where given }\end{array}$ \\
\hline $\mathrm{LiC}_{6}$ & Graphite \\
\hline $\mathrm{LiTiO}_{2}$ & \\
\hline $\mathrm{Li}_{2} \mathrm{Ti}_{5} \mathrm{O}_{12}$ & LTO \\
\hline
\end{tabular}

